\title{
Non-parametric analysis of a single seismometric recording to obtain building dynamic parameters
}

\author{
Marco Mucciarelli $\left({ }^{1}\right)$ and Maria Rosaria Gallipoli $\left({ }^{1}\right)\left({ }^{2}\right)$ \\ (') Dipartimento di Strutture, Geotecnica, Geologia Applicata all'Ingegneria (DiSGG), \\ Facoltà di Ingegneria, Università degli Studi della Basilicata, Potenza, Italy \\ ${ }^{(}{ }^{2}$ Istituto di Metodologie per l'Analisi Ambientale (IMAA), CNR, Tito Scalo (PZ), Italy
}

\begin{abstract}
We propose a simple time-domain, non-parametric method to estimate the damping at the fundamental frequency of a building. The method aims at obtaining quick-and-dirty data on large sets of buildings, at the expenses of the accuracy provided by other, more complex and resource-demanding techniques. The analysis of a $10 \mathrm{~min}$ recording of ambient vibration with a single high-resolution seismometer atop the building can provide a good estimate of the required parameters for the first flexural modes on orthogonal components. The proposed methodology does not require complex filtering and assumptions on signal structure, nor multiple measurement points or clear single transients induced by shakers, shocks or release tests. We checked the stability of the proposed method in terms of duration and characteristics of the signal, and compared the results obtained by others with standard techniques. Then, we tested the ability of the proposed technique to identify damping and frequency variations due to large displacements, damage or changes in the structural characteristics. The proposed methodology provides a satisfactory agreement when compared with other techniques. Even if it is not always possible to obtain higher modes, the advantage is that it is possible to study with limited resources the fundamental parameters for a large number of buildings. This is useful to include experimental data on building behaviour in microzonation studies.
\end{abstract}

Key words damping - fundamental mode - singledegree-of-freedom - ambient vibration

\section{Introduction}

The need for a simple and reliable method to estimate building damping arose during the project NATO Science for Peace 980857 (ASSAS$\mathrm{BV}$ ) whose aim is to mitigate seismic risk in the cities of Skopje, Zagreb and Ljubljana. One of

Mailing address: Dr. Marco Mucciarelli, Dipartimento di Strutture, Geotecnica, Geologia Applicata all'Ingegneria (DiSGG), Facoltà di Ingegneria, Università degli Studi della Basilicata, Viale dell'Ateneo Lucano 10, 85100 Potenza Italy; e-mail: mucciarelli@unibas.it the tasks of the project is to perform estimates of the fundamental frequency of buildings and of underlying soils, in order to identify possible resonance phenomena. This resonance approach was recently introduced for microzonation studies of large cities (e.g., Granada, Spain; Navarro et al., 2004). Obtaining fundamental frequency and damping is the first step to validate vulnerability models of buildings. Standard and detailed approaches can be used only for a limited number of selected buildings: the challenge faced in the ASSASBV project was to obtain simple but reliable estimates of dynamic parameters for thousands of building in a two-year time. This has to be achieved with the same instrumentation used on soils and without induced forcing.

The proposed methodology does not require filtering, pre-selection or assumptions on signal 
structure, nor multiple measurement points or clear single transients induced by shakers, shocks or release test. This overcomes some of the operational disadvantages of well established approaches like RandomDec (Vandiver et al., 1982), half-power method (Clough and Penzien, 1975), cross-correlation or wavelet-logarithmic decrement (Lamarque et al., 2000). For a detailed review of pros and cons of different ambient and forced vibration tests see Huang et al. (1999, and references therein), that also present a method applicable to multiple measurement points.

A generally accepted assumption is that the first approximation of the dynamic behaviour of a building is provided by the Single-DegreeOf-Freedom damped oscillator (SDOF)

$$
\ddot{x}+2 \gamma \dot{x}+\omega_{0}^{2} x=f(t)
$$

where $\gamma$ is the damping and $\omega_{0}$ is the fundamental frequency, in its turn related to the mass $m$ and stiffness $k$ of the system as

$$
\omega_{0}=\sqrt{\frac{k}{m}} .
$$

Even with this simple formulation, the system identification becomes a problem when the forcing term $f(t)$ is unknown. With ambient vibration excitation, a further warning comes from the difficulty to separate the noise filtered from the structure from the instrumental noise that is unavoidable during the measurements. The same problem is well known from microtremor measurements on soils, see e.g., Mucciarelli (1998) and Mucciarelli et al. (2005).

The solution of eq. (1.1) is given by

$$
x(t)=x_{0} e^{-\gamma t} \cos (\omega t+\varphi)+\varepsilon
$$

where $x_{0}$ is the initial amplitude, $\varphi$ the phase and $\varepsilon$ a perturbation term.

The damping is commonly derived as the logarithmic decrement of the amplitude over a pseudo-period $T$ according to the following equation:

$$
\gamma=\frac{1}{T} \ln |x(t) / x(t+T)| .
$$

Now we face the problem of numerical estima- tion of the above parameters from a discrete time series. If some form of excitation is applied, the damping can be straightforwardly estimated picking the amplitudes of subsequent maxima soon after the excitation is stopped and the system returns to its equilibrium position. For ambient vibration recordings, the RandomDec approach solves this problem using a stacking of pre-selected segments exceeding a fixed amplitude threshold $x^{\prime}$ with common initial conditions $\left(x(t)=x^{\prime} ; x(t+\Delta t)-x(t)>0\right)$.

\section{Proposed method and numerical simulations}

Assuming that the ground ambient vibration is close to white noise, a simple idealisation of the ambient vibration recording atop a building is composed by three factors

$$
X(\omega)=x_{\omega 0}(\omega)+x_{\varepsilon g}(\omega)+x_{\varepsilon i}(\omega)
$$

where $x_{\omega 0}(\omega)$ is the oscillation associated to the fundamental period (or $x(t)$ in eq. (1.3)), that cannot be observed in its full development because of the superposition of $x_{\varepsilon g}(\omega)$ that is the perturbation induced by the unknown, continuous forcing induced by ground ambient vibration (or $\varepsilon$ in eq. (1.3)). The term $x_{\varepsilon i}(\omega)$ represents the effect of instrumental noise during the measurement, as well as other sources of noise unfiltered by the building (e.g., wind impacting on unprotected sensors on a terrace roof). If we suppose that $x_{\omega 0}(\omega)>x_{\varepsilon g}(\omega)$ and $x_{\omega 0}(\omega)>>$ $>>x_{\varepsilon i}(\omega)$, then we can try to retrieve $\gamma$ directly from the recordings following this procedure:

i) Use a high resolution seismometer for taking measurements of ambient noise time histories. Accelerometers provide insufficient resolving power, as shown for measurements on soil by Mucciarelli (1998) and confirmed recently by the SESAME project (Duval et al., 2004).

ii) After a standard correction procedure (baseline correction and high-pass filtering), integrate the time-history to obtain displacements.

iii) Select only positive values that are local maxima. Build a $2 \times m$ matrix: amplitudes $x\left(t_{i}\right)$ of maximum in one column and time of occurrence $t_{i}$ in the other. 


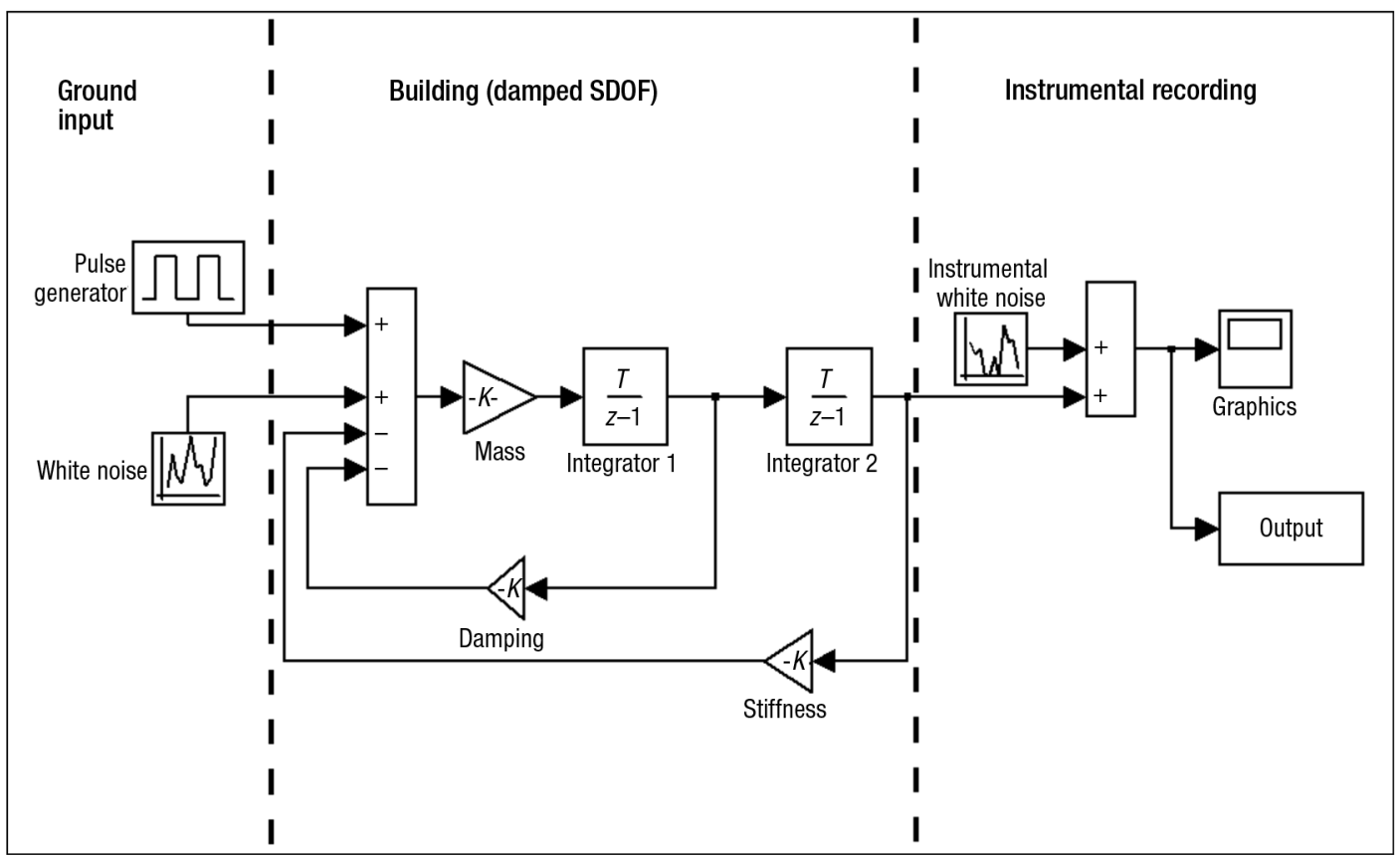

Fig. 1. SimuLink block model implemented to verify the assumptions underlying the NonPaDAn method.

iv) For each subsequent row in the above matrix, verify if $x\left(t_{i}\right)>x\left(t_{i+1}\right)$; if yes, calculate piecewise pseudo-frequency and damping as

$$
\begin{gathered}
v_{1}=1 /\left(t_{i+1}-t_{i}\right) \\
\gamma_{i}=(1 / 2 \pi) \cdot\left(x\left(t_{i}\right) / x\left(t_{i+1}\right)\right) .
\end{gathered}
$$

v) Build a 2-histogram, binning piecewise pseudo-frequencies and damping.

vi) Produce a graphic output to visualise the maximum of the empirical distribution, that is the main result of this non-parametric damping analysis (hereafter NonPaDAn), providing the fundamental frequency and its associated damping.

We implemented the above procedure using MatLab: the relevant $m$-file is freely available for download at the address <http:IInato.gfz.hr>.

To verify the above procedure and hypothesis, we built a numerical model using SimuLink. Figure 1 shows the relevant block diagram. The input is the sum of repeated, large pulses and
Gaussian white noise with variable amplitude. The mass and stiffness of the building model are tuned to obtain a fundamental frequency equal to $3 \mathrm{~Hz}$. The damping is set to $3 \%$. Before the recording, more Gaussian white noise is added to simulate the presence of instrumental noise.

Figure 2 reports the output of different simulations with increasing noise, both at the base and at the top of the building. It is possible to see how the NonPaDAn method retrieves correctly both damping and frequency if the noise is absent (maximum is the black dot inside the red area). The increase of input noise leaves the frequency unchanged but makes the damping peak broader. Nevertheless, the maximum in the 2D histograms shows the correct values for damping and fundamental frequency. On the contrary, sparse, spurious pseudo-frequencies arise when we add instrumental noise, and the damping is not correctly preserved. This simulation shows that the initial hypothesis is correct: to obtain a reliable estimate it is necessary to keep at a minimum the instrumental noise. 

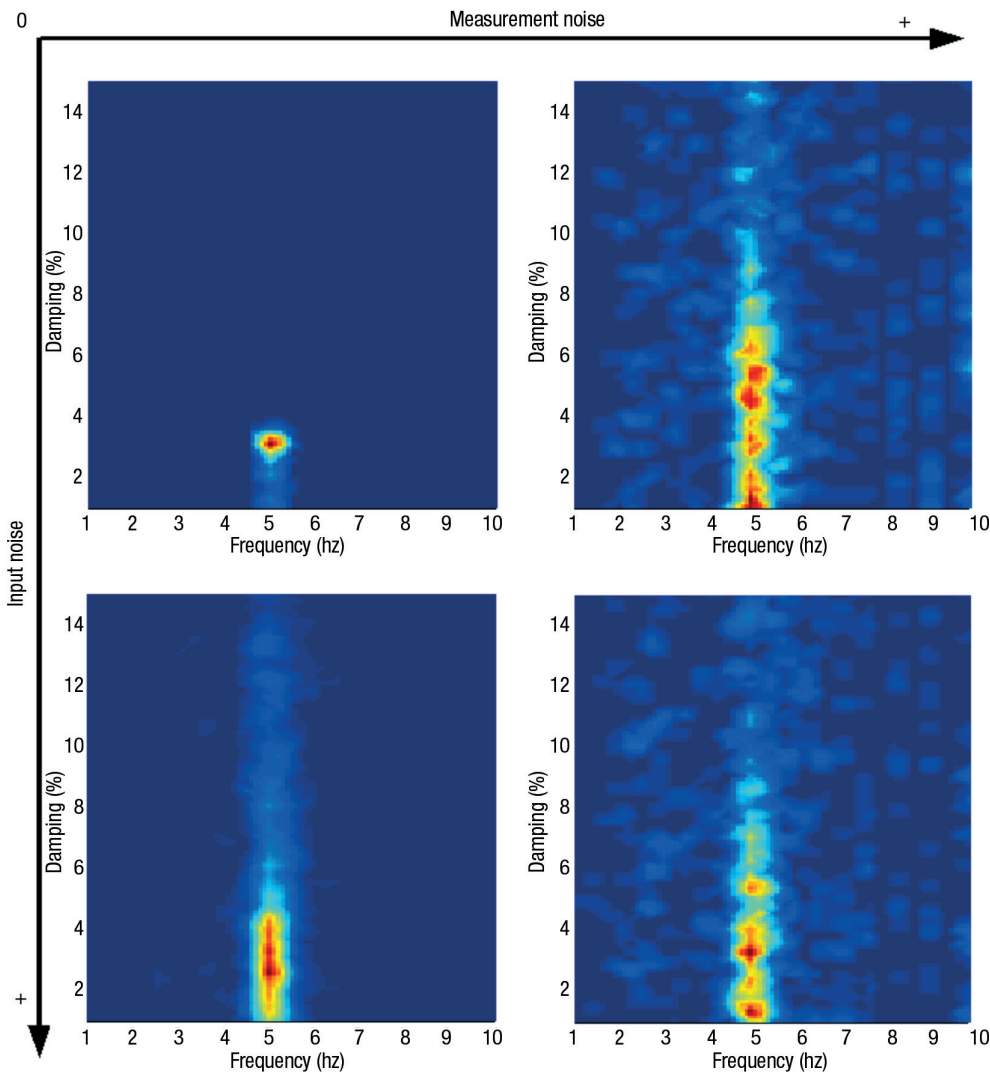

Fig. 2. Effects of increasing input and instrumental noise on the simulated recording atop a SDOF building.
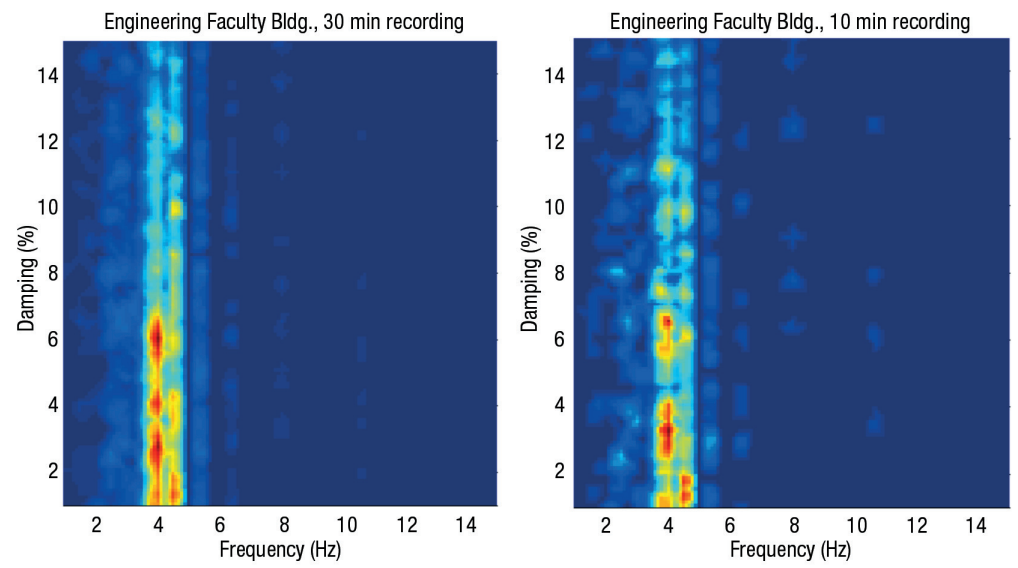

Fig. 3. NonPaDAn estimate of frequency and damping as a function of recording length (left: 30 min; right: 10 min). 


\section{Empirical data analysis}

The first check performed on the reliability of NonPaDAn is relevant to the duration of the signal. If using RandomDec, a minimum of $30 \mathrm{~min}$ of recording is required to obtain a stable estimate, according to Dunand et al. (2002). A 30 min recording at the 5th floor of the Engineering Faculty building at the University of Basilicata was performed using a digital tromometer (Micromed Tromino). Then, the damping was estimated using both the whole record and a section of $10 \mathrm{~min}$. Figure 3 shows the comparison: the fundamental frequency is the same and the damping remains centred at around $3 \%$. In this case it is also possible to identify a higher mode, but one can see in the following that it is not always so.

The second check was about the comparison with published results obtained using different methods to estimate frequency and damping.

Di Giulio et al. (2005) used the Horizontal to Vertical Spectral Ratio (HVSR) and the Hor-

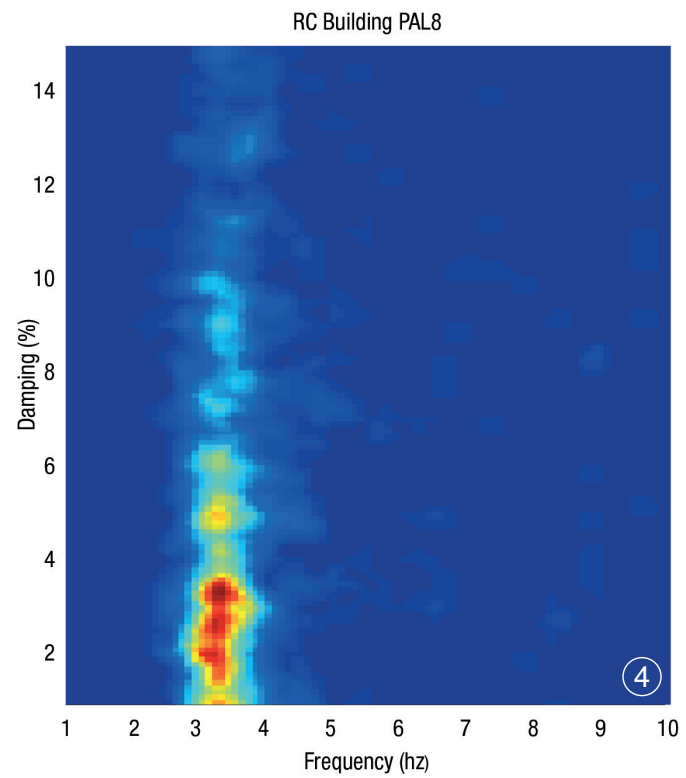

izontal to Horizontal Spectral Ratio (HHSR) technique to estimate the fundamental frequency of some buildings in the town of Palermo, Italy. The recordings were collected with a Lennartz 5 s seismometer and a MarsLite digitiser. We analysed 30 min of ambient vibration they recorded at the 5th floor of the R.C. building code-named PAL8. Figure 4 shows the result, with a clear estimate of fundamental frequency $(3.2 \mathrm{~Hz})$ and damping (3.5\%). The estimation of frequency is in a good agreement with the result given by Di Giulio et al. (2005).

During 2005 a series of tests were conducted on a R.C. scale model on the ENEA shaking table at the Casaccia (Rome) facility to study retrofit systems (Cardone et al., 2003). The input ranged from random noise to real strong motion. Cardone et al. (2005) estimated from clear transients a damping around $3 \%$ and a fundamental frequency of about $2 \mathrm{~Hz}$. Figure 5 reports the results obtained with NonPaDAn, in good agreement with the estimate from Cardone et al. (2005).

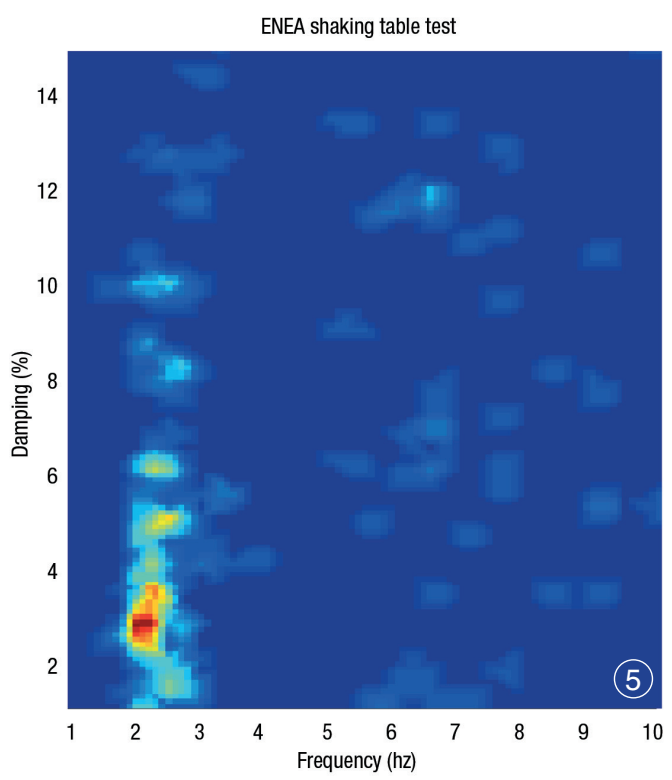

Fig. 4. NonPaDAn estimate of frequency and damping for a R.C. building (PAL8 in Di Giulio et al., 2004).

Fig. 5. NonPaDAn estimate of frequency and damping for a scale model tested with random vibration at the ENEA Casaccia shaking table (Rome). 
The last check is relevant to the ability of NonPaDAn to estimate the variations of frequency and damping due to large displacements, damage or changes in the structural characteristics.

For the first two items, the recordings obtained during the 2002 Molise earthquake sequence inside a building in Bonefro, Italy are re-analysed. The record comprises $1 \mathrm{~min}$ of ambient vibration, the 1 st November shock $\left(M_{w}=\right.$ $=5.6$ ) that damaged the building and whose vibration lasted $2.5 \mathrm{~min}$ and finally $1 \mathrm{~min}$ of ambient vibration. Mucciarelli et al. (2004) analysed this records using different techniques (Gabor transform, wavelets, moving window HVSR). The building has an initial frequency of $2.5 \mathrm{~Hz}$. During the large oscillations and subsequent damage the frequency lowers to $1.5 \mathrm{~Hz}$, to reach a final value for the damaged building at about 2 Hz. Figure 6 reports the result of NonPaDAn obtained for three 1 min windows: the first minute of ambient vibration, the coda of the shock and the last minute of ambient vibration. The frequency shift estimated with NonPaDAn agrees with the result of Mucciarelli et al. (2004). Moreover, it is worth noting that during the damaging large oscillations, a secondary, isolated damping emerges with a value of $10 \%$. This is
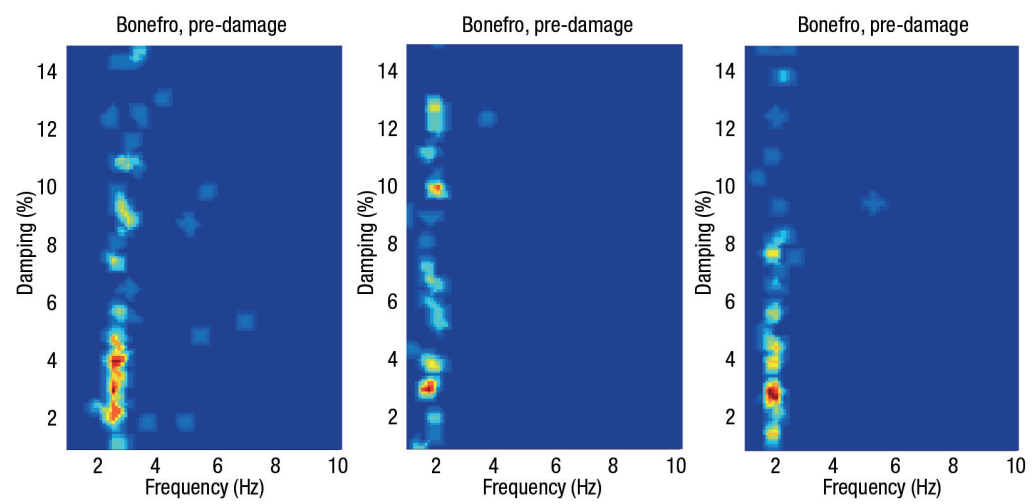

Fig. 6. NonPaDAn estimate of frequency and damping for a R.C. building in Bonefro, Italy, during the damage induced by the 1st November 2002 Molise earthquake.
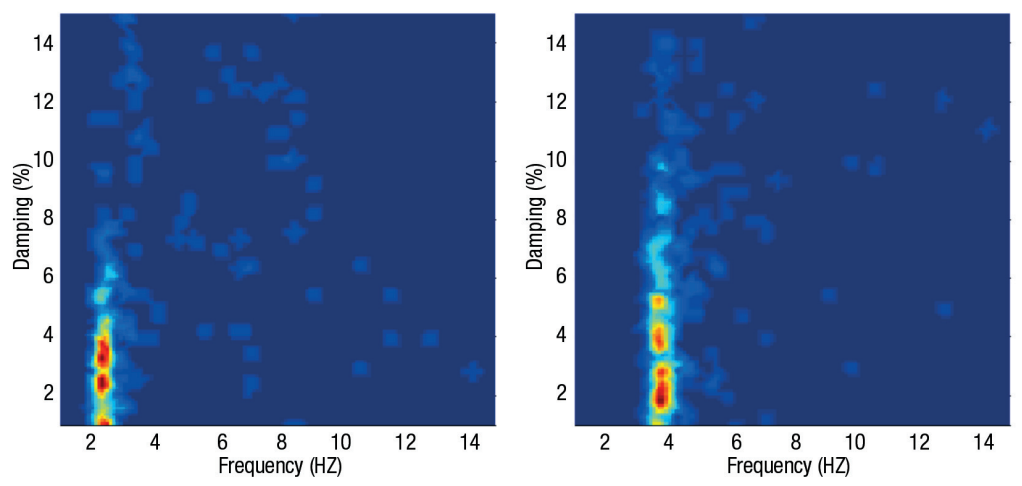

Fig. 7. NonPaDAn estimate of frequency and damping for a 4 stories R.C. Building in potenza, Italy with the bare frame only $(l e f t)$ and completed with infills (right). 
2.5 times the starting damping, in agreement with observation on damage in laboratory tests (see for example Cardone et al., 2004; and Dolce et al., 2004). The estimated damping factors are confirmed by a numerical modelling of the building, as described in Dolce et al. (2007).

The reliability of NonPaDAn to estimate the damping variation due to different building characteristics was checked taking advantage of a 4 storey, R.C. building under construction in Potenza, Italy. The first measurement was taken when the bare frame was completed and the bricks for the infills were loaded at each floor. The second measurement took place after the infills were completed. In this way, a variation of the stiffness occurred while the total mass of the building remained constant. In both case $10 \mathrm{~min}$ of ambient vibration were sampled with a Micromed digital tromometer. Figure 7 shows that NonPaDAn is able to capture the increase of frequency and the decrease of damping that was expected as a consequence of the increase in stiffness.

Using this last example, we take the proposed method a step further, suggesting a possible parameterisation of the model and thus the possibility of performing quantitative statistical tests.

The best way of observing the true damping of a structure is after forcing, when the free oscillations' amplitude decreases. The ambient noise is a particular case of output-only, unknown-input system: the forcing is random in amplitude and phase, so the structure cannot fully develop its damped free oscillation. Nevertheless, an improved strategy is to look for damping on more than two relative maxima separated by the same pseudo-period. These consecutive, decreasing local maxima are the best approximation of damped free oscillation that ambient noise measurement can produce. Of course a compromise is needed, between the search for long sequences (that would provide the best, more stable result) and the fact that the number of available sequences decrease exponentially when increasing their length. If we select only the damping values calculated on three consecutive maxima, the result is less dispersed and the size of the sample still permits to estimate their statistical distribution, which is log-normal. For the R.C. building with and without infills the

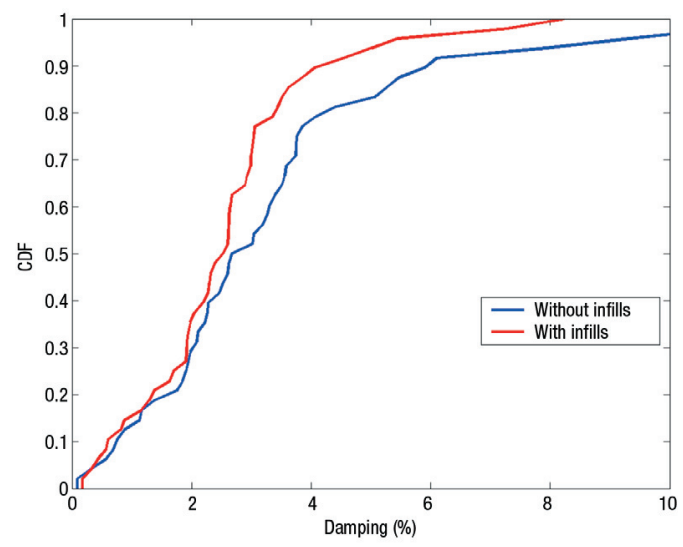

Fig. 8. Comparison of CDFs of damping derived by more than two subsequent maxima for the same building of fig. 7 .

plot of the two distributions (fig. 8) reveals that there is a constant shift of the damping by a factor 1.3. Moreover, a Kolmogorov-Smirnov test allows rejecting the hypothesis that the two CDFs derive from the same distribution with a $97.5 \%$ confidence level. This approach could be useful for measurements perfomed before and after an earthquake to establish if there is a significant frequency and damping shift due to damage: the ratio between CDFs appears to be more stable than the comparison between two couples of values.

\section{Conclusions}

We proposed a simple method for the nonparametric analysis of damping of buildings (NonPaDAn). It is able to capture the fundamental mode and its associated damping with a short, single measurement of ambient vibration atop a building. The resolving power is less than that provided by more advanced techniques and the higher modes and associated damping are rarely visible. However, the advantage of the proposed method is related to the limited resources it requires in terms of time, instrumentation and manpower. This candidates NonPaDAn as a tool to be used for dense measurement campaigns, when it is more important 
to know the fundamental dynamic parameters of large sets of buildings rather than focusing on the detailed behaviour of a single item.

\section{Acknowledgements}

This work was funded by NATO grant SfP 980857. Thanks are due to Antonio Rovelli and Giuseppe Di Giulio for providing recordings of PAL8, Felice Carlo Ponzo for providing recordings of ENEA test and Mauro Dolce for comments and suggestions.

\section{REFERENCES}

Cardone D., G. De Canio, M. Dolce, R. Marnetto, C. Moroni, M. Nicoletti, D. Nigro, A. Pizzari, F.C. Ponzo, E. Renzi, G. SANTARsiero and D. Spina (2003): Comparison of different passive control techniques through shaking table tests, in Proceedings of the 8th World Seminar on Seismic Isolation, Energy Dissipation and Active Vibration Control of Structures, October 6-10, 2003, Yerevan, Armenia.

Cardone, D., E. Coelho, M. Dolce and F. Ponzo (2004): Experimental behaviour of R/C frames retrofitted with dissipating and re-centring braces, J. Earthq. Eng., 8 (3), 361-396.

Cardone, D., A. Di Cesare, M. Dolce, C. Moroni, D. NiGRO, F.C. POnzo and M. Nicoletti (2005): Comparison of shaking table and pseudodynamic test on a base isolated R/C structure, in Proceedings of the 9th World Seminar on Seismic Isolation, Energy Dissipation and Active Vibration Control of Structures, June 13-16, 2005, Kobe, Japan.

Clough, R.W. and J. Penzien (1975): Dynamics of Structures (Mac Graw-Hill, New York), 2nd edition, pp. 576.

Di Giulio, G., R M. Azzara, G. Cultrera, M.S. GiAMmARINARo, P. VAllone and A. Rovelli (2005): Effect of local geology on ground motion in the city of Palermo, Italy, as inferred from aftershocks of the 6 September 2002 Mw 5.9 earthquake, Bull. Seismol. Soc. Am., 95, 2328-2341.

Dolce, M., D. Cardone, F.C. Ponzo and C. Valente (2004): Shaking table tests on reinforced concrete frames without and with passive control systems, Earthq. Eng. Struct. Dyn., 34, 1687-1717.
Dolce, M., F.C. Ponzo, A. Masi, M. Mucciarelli, M.R. Gallipoli, A. Di Cesare, M. Tetta and M. VonA (2007): Identification of the structural model and analysis of the global seismic behaviour of the IACP Bonefro building, Bull. Earthq. Eng. (submitted).

Dunand, F., P.-Y. Bard, J. L. Chatelain, Ph. Guéguen, T. VASSAIL and M.N. FARSI (2002): Damping and frequency from RandomDec method applied to in situ measurement of ambient vibrations: evidence for effective soil structure interaction, in Proceedings of the 12th European Conference on Earth Engineering, London, U.K., CD-ROM edition, Pap. no. 869.

Duval, A.-M., J.-L. Chatelain, B. Guillier and SESAME PROJECT WP02 TEAM (2004): Influence of experimental conditions on $\mathrm{H} / \mathrm{V}$ determination using ambient vibrations (noise), in Proceedings of the 13th World Conference on Earthquake Engineering, August 1-6, 2004, Vancouver, B.C., Canada, Pap. no. 306.

HuAng, C.S., Y.B. YANG, L.Y. Lu and C.H. Chen (1999): Dynamic testing and system identification of a multi-span highway bridge, Earthq. Eng. Struct. Dyn., 28, 857-878.

Lamarque, C.-H., S. Pernot and A. Cuer (2000): Damping identification in multi-degree-of-freedom systems via a wavelet-logarithmic decrement, Part 1 . Theory, $J$. Sound Vib., 235, 361-374.

MucCIARELl, M. (1998): Reliability and applicability range of the Nakamura's technique, J. Earthq. Eng., 2 (4), 625638.

Mucciarelli, M., A. Masi, M.R. Gallipoli, P. HarabaGLiA, M. VonA, F. Ponzo and M. DolCE (2004): Analysis of a R.C. building dynamic response and soil-building resonance based on data recorded during a damaging earthquake (Molise, Italy 2002), Bull. Seismol. Soc. Am., 94 (5), 1943-1953.

Mucciarelli, M., M. R. Gallipoli, D. Di Giacomo, F. Di NotA and E. Nino (2005): Wind influence on seismic noise measurements, Geophys. J. Int., 161, 303-308.

Navarro, M., F. Vidal, M. Feriche, T. Enomoto, F.J. SÁNCHEZ and I. MATSUDA (2004): Expected ground-RC building structures resonance phenomena in Granada city (Southern Spain), in Proceedings of the 13th World Conference on Earthquake Engineering, August 1-6, 2004, Vancouver, B.C., Canada, Pap. no. 3308.

Vandiver, J.K., A.B. Dunwoody, R.B. CAmpbell and M F. CooK (1982): A mathematical basis for the random decrement vibration signature analysis technique, $J$. Mech. Des., 104, 307-313.

(received October 27, 2006; accepted April 4, 2007) 